\title{
Special-purpose computer with highly parallel pipelines for solution $X$-ray scattering analysis of macromolecules XSAM-2
}

\author{
Satoru Hosono ${ }^{\text {a) }}$, Hideaki Wakabayashi, Nobuyuki Masuda, \\ and Tomoyoshi Ito \\ Graduate School of Engineering, Chiba University, \\ 1-33 Yayoi-cho, Inage-ku, Chiba 263-8522, Japan \\ a) satori@graduate.chiba-u.jp
}

\begin{abstract}
We have developed a special-purpose computer for analyzing X-ray scattering of models of macromolecules, named XSAM-2 (solution X-ray Scattering Analysis of Macromolecules). XSAM-2 contains 64 circuits operating in parallel for calculating X-ray scattering intensities using the pipeline method. XSAM-2 calculates 122 times faster than a personal computer with a Pentium 4 processor.
\end{abstract}

Keywords: hardware, special-purpose computer, solution X-ray scattering, biological macromolecule

Classification: Science and engineering for electronics

\section{References}

[1] O. Glatter and O. Kratky, in O. Glatter and O. Kratky (Editors), "Small Angle X-ray Scattering," Academic Press, New York, 1982.

[2] M. Arai, K. Ito, K. Maki, T. Ikura, T. Inobe, H. Kihara, Y. Amemiya, and K. Kuwajima, "Structural analysis of protein folding intermediates by solution X-ray scattering," In Old and New Views of Protein Folding, Amsterdam, Elsevier Science, pp. 31-40, 1999.

[3] K. Soda, Y. Miki, T. Nishizawa, and Y. Seki, "New method for incorporating solvent influence into the evaluation of X-ray scattering of proteins in solution," Biophys. Chem., vol. 65, pp. 45-53, 1997.

[4] I. Pitz, E. Schwarz, T. Suzuki, and T. Gotoh, "Small-angle X-ray scattering studies of giant haemoglobin from the annelid Tylorrhynchus heterochaetus," Int. J. boil. Macromol., vol. 10, pp. 356-360, 1988.

[5] Y. Igarashi, K. Kimura, K. Ichimura, S. Matsuzaki, T. Ikura, K. Kuwajima, and H. Kihara, "Solution X-ray scattering study on the chaperonin GroEL from Escherichia coli," Biophys. Chem., vol. 53, pp. 259-266, 1995.

[6] S. Hosono, H. Wakabayashi, N. Masuda, and T. Ito, "Special-purpose computer for solution X-ray scattering analysis of macromolecule," IEICE Trans. Inf. E Syst., vol. J90-D, no. 2, pp. 399-405, 2007 (in Japanese).

[7] Y. Seki, T. Tomizawa, N. N. Khechinasvili, and K. Soda, "Contribution of solvent water to the solution X-ray scattering profile of proteins," Biophys. Chem., vol. 95, pp. 235-252, 2002. 
[8] T. Ito, N. Masuda, K. Yoshimura, A. Shiraki, T. Shimobaba, and T. Sugie, "A special-purpose computer HORN-5 for a real-time electroholography," Optics Express, vol. 13, no. 6, pp. 1923-1932, 2005.

\section{Introduction}

In recent years, it has become possible to predict the three-dimensional structure of biological macromolecules, because of the progress in experimental techniques (for example, X-ray diffraction), simulation methods of pattern matching of Molecular Dynamics (MD), homology searches, and so on. Biological macromolecules normally occur in solution, and direct identification of the three-dimensional structure of biological macromolecules in solution remains a difficult problem.

Experimentally, the solution X-ray scattering method, which makes use of a scattered wave, is one analytical method for obtaining the molecular structure of macromolecules in solution. Using the scattered wave, we can obtain certain information about molecules, including their interior radius, molecular mass and secondary structure $[1,2,3,4,5]$. Experimental devices for obtaining the X-ray scattering pattern are continually improving; for example it is now possible to obtain intensities for large scattering angles. It seems likely that in the future further three-dimensional structure information will become available.

The experimental scattered wave pattern is compared with the pattern produced from a structural model of the molecule [6]. Specifically, a likely model is constructed from information about the molecular structure obtained from homology search, a database, or MD. We then perform a numerical simulation to obtain the scattered pattern for the model. Next, the experimental scattered pattern is compared with the pattern obtained from the numerical simulation. The model is then modified, and the process is repeated until the pattern from the simulation matches the pattern from the experiment.

However, this method requires a high-speed calculating system, because the time to calculate the scattered pattern is proportional to the square of the number of atoms in the biological macromolecule and this number is very large. Additionally, in recent years, it has become necessary to consider models with molecules of water placed around the protein, in order to compare simulation results with experimental results for macromolecules in solution [7]. Therefore tens of thousands of particles are necessary to simulate a small protein.

The intensity of the scattering wave is given by

$$
I(h)=\sum_{j}^{N} \sum_{k}^{N} f_{j} f_{k} \frac{\sin \left(h r_{j k}\right)}{h r_{j k}}
$$

where $N$ is the number of particles in the molecule, $f_{j}$ is the scattering factor of particle $j$ and $r_{j k}$ is the distance between particles $j$ and $k$. The parameter 
$h$ is given by $h=4 \pi \sin (\theta) / \lambda$, where $2 \theta$ is the scattering angle and $\lambda$ is the wavelength of the X-ray. Equation (1) can also be applied to water molecules. Most of the calculation time in the simulation is taken up with the numerical solution of Eq. (1).

To speed-up the calculation, we developed hardware, named XSAM-1, to numerically solve Eq. (1). The calculation speed of XSAM-1 was 2.3 times faster than that of a personal computer (PC) with a Pentium 4 CPU, 2 GB of memory and running at $3.2 \mathrm{GHz}$. This showed that XSAM-1 was effective for numerical calculation of the solution X-ray scattering [6].

$\mathrm{XSAM}-2$ is a machine developed from XSAM-1 to make the latter more practical. It is a special-purpose computer system containing 64 circuits of XSAM-1, operating in parallel. The calculation speed of XSAM-2 is about 100 times faster than that of a PC.

In Section 2 we describe the hardware design of XSAM-2, and in Section 3 we describe its performance. In Section 4, we discuss the results.

\section{Hardware Design of XSAM-2}

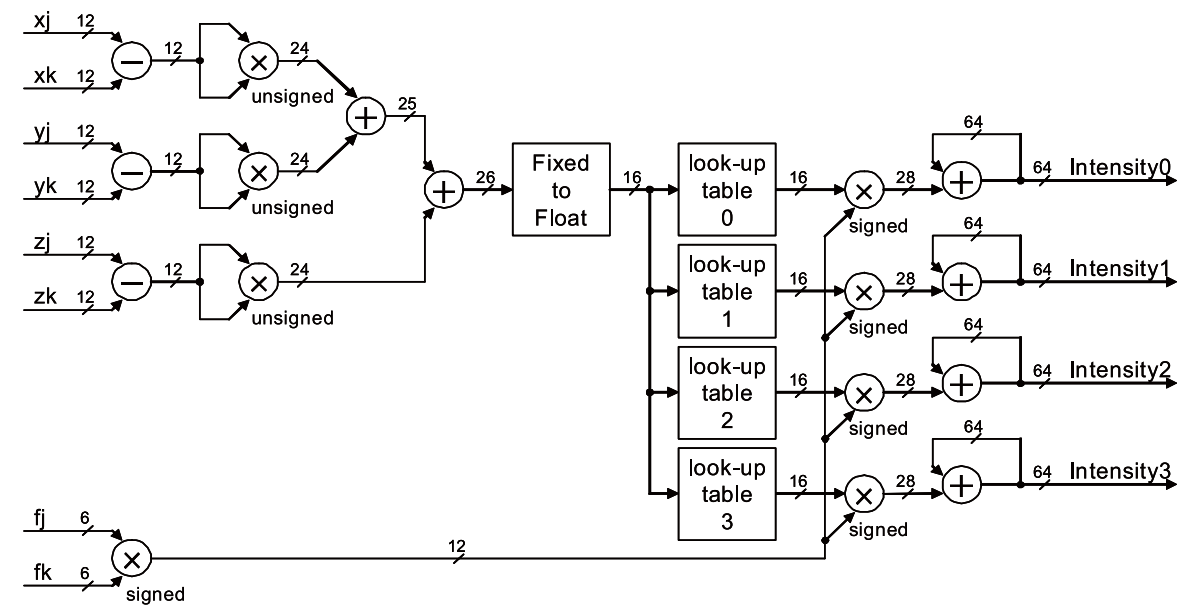

Fig. 1. Block diagram of four pipelines in parallel on one chip.

In Eq. (1), $h$ depends upon the scattering angle. Calculation is carried out for 500 values of $h$. For a given value of $h$, a pipeline of XSAM-2 can evaluate Eq. (1) independently. Therefore the pipelines can work in parallel.

Figure 1 shows the basic circuit of XSAM-2. Following Eq. (1), the hardware calculates $\sin (h r) / h r$, after calculation of the distance $r_{j k}$. In XSAM-2, look-up tables are used for this. It is possible to calculate in parallel by preparing some look-up tables for different values of $h$. Figure 1 shows four look-up tables prepared; namely look-up tables $0,1,2$, and 3 for $h_{0}, h_{1}, h_{2}$, and $h_{3}$, respectively.

In Fig. 1, the calculations standing in line vertically are taken out simultaneously at each clock. The circuit consists of eight pipelined stages. Since Eq. (1) can be calculated independently on each $h$, the circuit of XSAM-2 
can have several pipelines after the stage of "Fixed to Float". The output of XSAM-1 was only one (Intensity 0 ) since we could make the circuit simple. In XSAM-2, we improved the circuit design to output four results (Intensity $0,1,2$ and 3) simultaneously on one chip.

The number of pipelines (outputs) in parallel is limited by the gate size of the Field Programmable Gate Array (FPGA). We developed XSAM-2 using a large-scale FPGA board made by our study group [8]. On this board, four FPGA chips were mounted. Each FPGA chip was a Xilinx Virtex II Pro XC2VP70, with 7 million gates equivalent (33,088 slices of the Xilinx standard). The board was designed in accordance with the peripheral component interconnect (PCI) standard and so could be connected to a PC using the PCI slot.

We implemented the circuit in Fig. 1 into each FPGA chip. The possible number of parallel pipelines (outputs) depends on the size of the RAM in the FPGA chip. The size of the inside RAM is 5,904 Kbits. It also limits the width of input data. We adopted 12 bits for the data width due to the limitation.

Since each board held four FPGA chips, it was possible to implement four copies of the circuit in Fig. 1 into one board. We were able to install several boards in the PC, because this board was designed according to the PCI standard. We constructed XSAM-2 with four large-scale FPGA boards on one personal computer. XSAM-2 is thus a special-purpose computer with a highly parallel pipeline system, which contains 16 units as in Fig. 1, each able to calculate simultaneously and independently, providing 64 intensities in total. The working frequency was $181.5 \mathrm{MHz}$.

\section{Performance of XSAM-2}

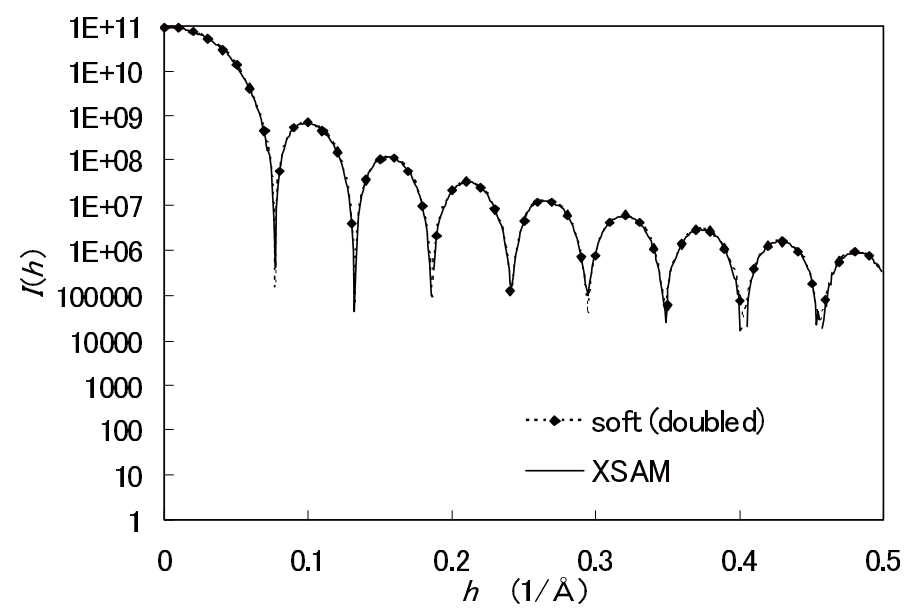

Fig. 2. Block diagram of four pipelines in parallel on one chip.

As a test to evaluate the performance of XSAM-2, we simulated the intensity of the scattering wave from 30,976 spherical crossly distributed particles, 
for 500 values of $h$. We carried out the same simulation using a PC, for comparison.

In Fig. 2, the dotted line shows the result of calculation by PC and the solid line shows the result calculated by XSAM. The result of calculation by XSAM agrees closely with that of double precision calculation by the PC.

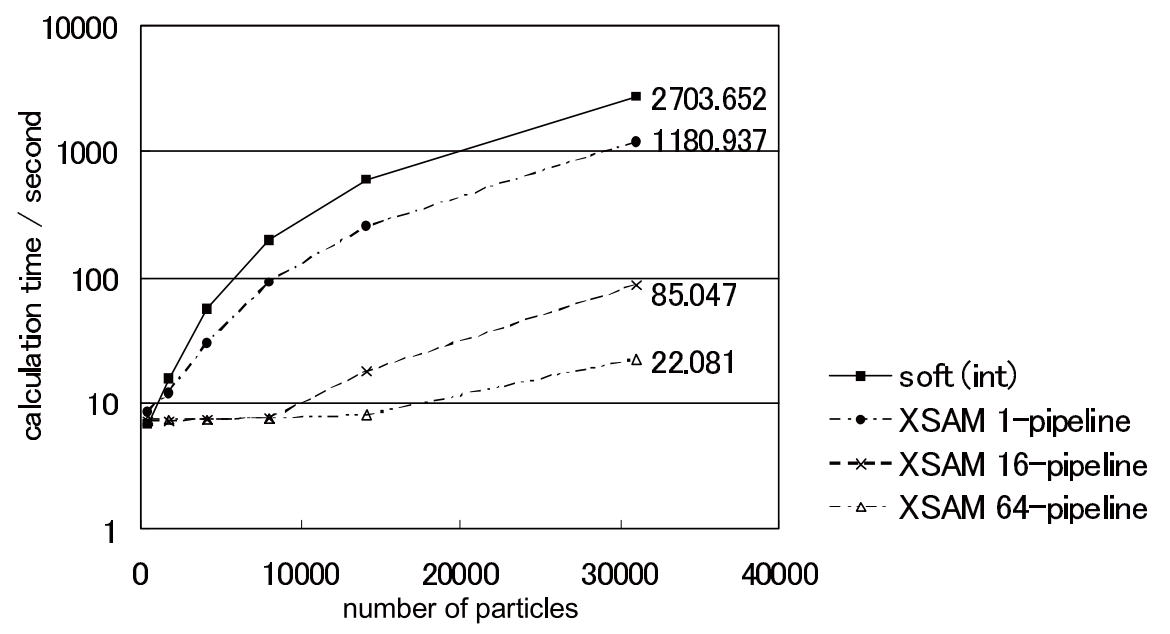

Fig. 3. Block diagram of four pipelines in parallel on one chip.

In Fig. 3, we compare the calculation times of a single pipeline system in hardware (equivalent to XSAM-1), a hardware system with 16 parallel pipelines in parallel (the one board system) and 64 parallel pipelines (XSAM2), with that of the PC.

In Fig. 3, the calculation time using only integer calculation by the PC is shown, so that the calculation precision of the $\mathrm{PC}$ is matched with that of the hardware. The double precision calculation, which was used to evaluate the precision of XSAM in Fig. 2, is more than 10 times slower than the integer calculation.

In the XSAM system, the preparation of look-up tables for each $h$ by the host computer before calculation is required. The preparation of look-up tables for each $h$ does not depend on the number of pipelines in parallel, but it does depend on the subdivision of $h$. For 500 values of $h$, the preparation of look-up tables takes 6.5 seconds. It takes 1.5 seconds to transfer the table from the host PC to the FPGA board. Therefore it costs about 8 seconds to prepare look-up tables, independently of the number of particles, $N$. From this, the calculation times of the system of 16 pipelines and 64 pipelines in Fig. 3 are approximately 8 seconds when $N$ is small or the calculation time is short.

The special-purpose computer becomes necessary as $N$ becomes large, when the calculation time for the PC becomes impractical. The calculation speed increases for large $N$ as more pipelines are used in parallel. On the test simulation with 30,976 particles, taking the speed of the PC to be 1 , the ratio of speed-up is 2.3 times with 1 pipeline, 32 times with 16 pipelines 
and 122 times with 64 pipelines. The ratio of speed-up is proportional to the number of parallel pipelines.

\section{Conclusion}

The purpose of this system is to find the structure of (non-crystalline) biological macromolecules in solution, which involves repeatedly revising a molecular model. The advantage of this system is that we can compare directly the result of experiment with the result of numerical simulation. As a result we expect to be able to investigate the quality and function of actual biological macromolecules.

We revised XSAM-1 to construct XSAM-2 with highly parallel pipelines. The resulting calculation speed of XSAM-2 was about 50 times faster than that of XSAM-1 and more than 100 times faster than a PC. In the case of numerical calculation with about 30,000 particles, the calculation time of XSAM-2 was 22 seconds and that of the PC was 2,703 seconds (approximately 45 minutes).

In future work, we plan to construct XSAM-2 clusters and thereby further speed-up XSAM, thus making it possible to construct a practical system to evaluate experimental field data on macromolecules. 